\title{
PLURALISMO DE LOS VALORES, NUEVO CONSTITUCIONALISMO Y BALANCE LIBERTAD-SEGURIDAD *
}

\author{
Mauro Barberis \\ Universidad de Trieste ${ }^{* *}$ \\ Idon'tunderstand why the strongestnationson Earth believes that \\ acknowledging a mistake will threaten its security
}

(K. AL-MASRI)

RESUMEN. Después del 11 septiembre de 2001, se habla cada vez más de supuestos balances entre libertad y seguridad, balances que corren el riesgo de convertir el Estado constitucional en Estado de seguridad (Security State). La metáfora del balance es compartida entre el value pluralism, la metaética que atribuye a los valores características como objetividad, pluralidad, conflictividad, inconmensurabilidad e indeterminación (apartado 2), y el new constitutionalism de la posguerra, que atribuye las mismas características a los principios constitucionales (apartado 3). Sin embargo, el análisis de los supuestos balances entre libertad, por un lado, y seguridad individual, social y nacional, por el otro (apartado 4), produce como resultado que restringir libertades individuales disminuye también la seguridad individual, sin aumentar la seguridad colectiva en algún grado significativo.

Palabras clave: pluralismo de los valores, nuevo constitucionalismo, libertad, seguridad.

\section{Pluralism of values, new constitutionalism and freedom-security balancing}

ABSTRACT. After $9 / 11$, is increasing talk of alleged balances between liberty and security - balances foreshadowing the transformation of Constitutional State in a Security State. The balance's metaphor is shared by value pluralism, the metaethics ascribing to values characters like objectivity, plurality, conflictivity, incommensurability, and indeterminacy (§ 2), with postwar new constitutionalism, ascribing the same characters to constitutional principles (§3). However, the analysis of the alleged balances between liberty, on the one hand, and individual, social and national security respectively, on the other, produces the following results. Restraining individual liberties weakens individual security too, without increasing at a significant extent collective security.

Keywords: value pluralism, new constitutionalism, liberty, security.

\footnotetext{
* Fecha de recepción: 20 de septiembre de 2016. Fecha de aceptación: 20 de octubre de 2016.

Este trabajo amplía las lecciones del Master en Global Rule of Law and Constitutional Democracy (Imperia, 10 de febrero de 2016), y ha sido financiado por los fondos FRA 2014 de la Universidad de Trieste.

** Profesor Ordinario de Filosofía del Derecho en la Universidad de Trieste. Mail: barberis@units.it.
} 


\section{PREMISA}

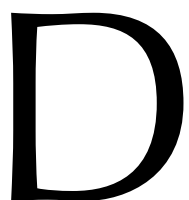

esde el 11 de septiembre, al menos, se habla obsesivamente de balancear libertad y seguridad. La metáfora de la balanza es común al pluralismo valorativo (value pluralism), la metaética que predica la objetividad, pluralidad, conflictividad e inconmensurabilidad de los valores éticos o prácticos (apartado 2), y al nuevo constitucionalismo postbélico (new constitutionalism), que predica esas mismas características de los principios constitucionales (apartado 3). No obstante, un análisis del balance entre libertad, de un lado, y seguridad individual, social y nacional, del otro (apartado 4), genera esta conclusión: restringir la libertad disminuye la propia seguridad individual, sin aumentar en alguna medida apreciable la seguridad colectiva.

\section{PLURALISMO DE LOS VALORES}

El pluralismo de los valores no es una ética normativa, más o menos liberal ${ }^{1}$. Si lo fuese, no constituiría un terreno adecuado para hablar de los conflictos entre libertad y seguridad y de los posibles balances entre ellas. En efecto, si fuese una ética normativa como las teorías de la justicia liberal o libertaria, consideraría a la libertad como el valor último, y rechazaría la idea de su balance con la seguridad, como un artificio retórico. Este trabajo llega a conclusiones no muy diferentes pero tomando en serio los argumentos por la seguridad: en algún sentido es verdadero que esta pudiera prevalecer sobre la libertad.

El pluralismo de los valores es una metaética: una investigación sobre el estatus del desacuerdo en el discurso ético y/o en el razonamiento práctico ${ }^{2}$. La metaética presenta un desacuerdo ulterior, de según nivel, entre dos familias de posiciones que denominaré, apresuradamente, objetivismo y subjetivismo (meta)éticos ${ }^{3}$ ( $c f r$. 2.1). Adoptando la conocida distinción entre niveles intuitivos y críticos, respectivamente, del discurso ético y/o del razonamiento práctico ${ }^{4}$, las razones —empíricas o fácticas, analíticas o conceptuales, normativas o valorativas- en pro y en contra de tales posiciones podrían presentarse así.

Según el objetivismo metaético, los desacuerdos que se presentan comúnmente en las discusiones de ética normativa serían legítimos solo prima facie; a un nivel más profundo, all things considered, se revelarían como ilegítimos. En efecto, los problemas

1 Sobre este punto, vid. la discusión realizada en Political Studies, 42/2, 1994, entre George CrowDER y los dos mayores exponentes del value pluralism, Isaiah BERLIN y Bernard WILLIAMS. Un alumno de BERLIN, que asume posiciones de compromiso entre libertad y seguridad, es Michael IGNATIEFF: cfr. id., The Lesser Evil: Political Ethics en an Age of Terror, Princeton, Princeton, U. P., 2004.

${ }^{2}$ Los teóricos del derecho hablan de desacuerdos interpretativos: $c f r$. P. LuQue SÁnChez, G. B. RatTi (eds.), Acordes y desacuerdos. Cómo y por qué los juristas discrepan. Madrid, Marcial Pons, 2012. Los estudiosos de metaética deberían hablar de desacuerdos éticos o prácticos.

3 A pesar de las contraindicaciones, prefiero estas denominaciones, usadas también por los autores de los que me ocupo, a otras no menos comunes pero en absoluto coincidentes como cognoscitivismo/no-cognoscitivismo o, peor aún, realismo/antirealismo.

${ }^{4}$ La distinción, ampliamente retomada también por los teóricos del derecho, se debe a R. M. HARE, Moral Thinking: Its Levels, Methods, and Point, Oxford, Oxford U. P., 1981. 
ético-normativos, incluso solo para poderse decir tales, deberían siempre admitir una solución objetiva, o correcta, o mejor que otras. Según el subjetivismo metaético, en cambio, el desacuerdo prima facie podría permanecer perfectamente como legítimo incluso all things considered; después de todo, los problemas ético-normativos son tales precisamente porque admiten más respuestas legítimas, que siempre pueden ser puestas nuevamente en discusión.

Entre las diversas razones del desacuerdo metaético haría falta, quizás, atender mejor a los distintos objetivos de las disciplinas en cuestión. Para las disciplinas predominantemente normativas, como la filosofía moral o política, pero también, de un modo distinto, la economía o la doctrina jurídica, es esencial justificar soluciones compartidas; quizás no por casualidad muchos estudiosos de estas disciplinas son objetivistas. En cambio, para las disciplinas predominantemente cognoscitivas, como la teoría del derecho y las ciencias sociales es, al menos, igualmente importante explicar los desacuerdos; quizás también por esto muchos estudiosos de tales disciplinas son subjetivistas.

La discusión entre objetivistas y subjetivistas, como veremos, tiende a ser reemplazada por la discusión entre monistas y pluralistas de los valores. No obstante, para evitar equívocos, esta última debe ser distinguida de la discusión sobre la unidad o bien la fragmentación del razonamiento práctico. A menudo, los teóricos del derecho han sostenido la fragmentación del razonamiento práctico en moral, político, jurídico, excluyendo así la relevancia de los valores éticos para el razonamiento jurídico. En cambio, los valores se tornan esenciales para los partidarios de la unidad del razonamiento práctico: el estatus de los valores no es menos importante para los teóricos del derecho que para los filósofos morales o políticos 5 .

Finalmente, la distinción entre monismo y pluralismo de los valores, gira en torno al desacuerdo o, mejor dicho, al conflicto en la ética, y a su posible solución all things considered. No es, en cambio, una dicotomía o una clasificación en sentido lógico; es una oposición polar. Se dan, al menos, cinco alternativas a propósito de los valores: no solo objetividad/subjetividad, sino también unidad/pluralidad, armonía/conflicto, conmensurabilidad/inconmensurabilidad, determinación/ indeterminación. Según la elección que se tome en cada una de estas alternativas, cada autor se ubicará más cerca bien del polo monista o bien del polo pluralista.

Un desarrollo importante del pluralismo de los valores, y de sus relaciones con el derecho, ha sido provisto por Bruno CELANO. Cito un pasaje suyo, muchas de cuyas expresiones serán examinadas seguidamente.

El derecho es necesariamente expresión de valores y principios éticos objetivos. Es tarea del derecho positivo la determinación de estos principios y valores. Pero los valores, por hipótesis objetivos, son múltiples, conflictivos, inconmensurables e indeterminados. Su determinación exige, pues, ponderación, resolución de conflictos, conmesuración de lo inconmensurable, determinación de lo indeterminado. El derecho [positivo] realiza esta determinación mediante una estructura nomodinámica (instituciones de poderes normativos) ${ }^{6}$.

5 Debo esta distinción a una discusión con Nicola MufFATO.

6 B. Celano, derecho, justicia, razones. Madrid, Centro de Estudios Políticos y Constitucionales, 2009, 150. La traducción es de José Juan MORESO. 
En otra ocasión he presentado al pluralismo de los valores como la metaética del constitucionalismo ${ }^{7}$. Aquí, en cambio, en el apartado 2 , formulo cinco tesis distintivas del value pluralism sobre los valores éticos o prácticos, mientras que en el apartado 3 formulo cinco tesis correspondientes sobre los principios constitucionales emergentes en el new constitutionalism. Las correspondencias entre estas tesis no se deben a influencias recíprocas; normalmente, filósofos de la ética y constitucionalistas se ignoran recíprocamente. Más bien, los principios del nuevo constitucionalismo parecen ser la formulación jurídica de los valores del pluralismo, una suerte de equilibrio reflexivo en la que metaética e instituciones constitucionales se enriquecen recíprocamente.

\section{1. ¿Valores éticos objetivos?}

En metaética, la discusión entre objetivistas y subjetivistas tiende a ser reemplazada por aquella entre monistas y pluralistas de los valores, aun cuando, luego, no coincidan ambas distinciones. Ciertamente, los monistas (iusnaturalistas, utilitaristas...) son objetivistas, mientras que los padres del pluralismo, Friedrich NIETZSCHE y Max WEBER, son subjetivistas, más o menos radicales. Sin embargo, pluralistas como Celano, Isaiah BERLIN (el inventor de la propia etiqueta «value pluralism») y Bernard WILLIAMS, se declaran objetivistas, con una ambigüedad que hace falta disipar, aunque solo sea para mostrar en qué el pluralismo de los valores constituye un progreso en relación con el subjetivismo metaético.

La vieja disputa entre objetivistas y subjetivistas versa sobre los valores solo por metonimia; en realidad, versa sobre los juicios de valor, de los que los valores constituyen los parámetros. Pluralistas como BERLIN, WiLLIAMS y CELANO consideran los valores objetivos solo en tanto que dados en la cultura humana; como veremos más adelante (cfr. 3.1.), se podría pensar en ellos como el producto de procesos de abstracción. Es decir, que de juicios de valor más o menos subjetivos, por ejemplo, en términos de justicia, libertad, solidaridad, se abstraen valores objetivos como la justicia, la libertad, la solidaridad, pero con el precio de su indeterminación (pluralidad, conflictividad, inconmensurabilidad, además de su genericidad) ${ }^{8}$.

Por tanto, debiendo elegir, conciliar, conmensurar y determinar valores semejantes, los juicios de valor permanecen, en gran medida, como subjetivos. Precisamente por esto, sin embargo, el pluralismo de los valores constituye un progreso con respecto a las metaéticas subjetivistas. Ello, en efecto, explica-justifica el acuerdo sobre valores indeterminados $y$ el desacuerdo sobre sus determinaciones, identificando las razones del uno y del otro. En la formulación de CELANO, además, esta metaética pluralista

7 Cfr. M. BARBERIs, Etica per giuristi (2006), trad. cast. Ética para juristas, Madrid, Trotta, 2008, 163-197 y, desde el título, «Metaética del constitucionalismo», en Diritto \& questioni pubbliche, 2011, 133-156.

8 BERLIN implícitamente, CELANO explícitamente, invocan al respecto el principio de caridad interpretativa de Willard QUINE y Donald DAVIDSON; para comprender creencias ajenas debemos postular necesariamente alguna semejanza con las nuestras. Por ejemplo, I. BERLIN, «My Intellectual Path» (1998), trad. cast. «Mi trayectoria intelectual», en id., Dos conceptos de libertad y otros escritos, Madrid, Alianza, 2001, 123-136, se pregunta si del conjunto de los valores objetivos forman parte los «valores» nazis, y contesta que sí: podríamos comprender que mujeres y hombres como nosotros, bajo ciertas condiciones, hayan podido compartirlos. 
restituye un rol crucial al derecho al ser llamado, en la división del trabajo ético, a determinar valores objetivos pero, o porque, indeterminados? .

\section{2. ¿Valores éticos plurales?}

Que los valores sean múltiples sonaría obvio, prima facie, si no fuese posible, all things considered, reducirlos a la unidad. Es precisamente esto lo que hace al monismo de los valores, sostenido por BERLIN, la tradición central de la ética occidental: reduce la pluralidad de los valores a la unidad, o a un reducido núcleo de valores últimos. En su momento, la eudaimonia, el bien(sumo), el respeto de los pactos, la vida, la utilidad, la dignidad humana, fueron consideradas el valor último o, quizás, el ingrediente común a todos los valores que pueden fungir de criterio para solucionar los (aparentes) conflictos, o al menos de medida común para remediar su (aparente) inconmensurabilidad.

Todas las posiciones pluralistas, en sentido amplísimo, comparten la negación del monismo. Sin embargo, es necesario distinguir el significado de tres expresiones usadas a menudo para referirse a ellas: «polité́smo de los valores», «pluralismo de los valores» y «pluralismo ético». El politeísmo de los valores (Polytheismus der Werte), de Max WEBER, abarca todos los valores: religiosos (sacro/profano), epistémicos (verdadero/ falso), estéticos (bello/feo), y estrictamente éticos (bueno/malo) ${ }^{10}$. El value pluralism de BERLIN oscila, pero termina refiriéndose, sobre todo, a valores éticos o prácticos. El pluralismo ético o práctico, finalmente, se refiere exclusivamente a estos últimos.

Incluso, la elección entre monismo y pluralismo de los valores, como toda otra opción metaética, depende inescindiblemente de consideraciones de tres tipos: empíricas o fácticas, analíticas o conceptuales, normativas o valorativas. Siempre es posible intentar distinguir estos tres componentes en cualquier posición metaética y, a menudo, es necesario hacerlo, aunque es claro que están inextricablemente conectados. Si se les distingue - como tienden a hacer los filósofos analíticos, aun cuando problematizan las distinciones entre empírico, analítico y normativo- se puede hablar de metaética; en cambio, cuando no se les distingue, se puede hablar más propiamente de metafísica ${ }^{11}$.

\section{3. ¿Valores éticos conflictivos?}

¿Forman los valores un kosmos, un conjunto ordenado de bienes o, más bien, un caos de impulsos vitales conflictivos? El value pluralism asume que los valores son plurales, lo que no basta para definirlo. John FINNIS, por ejemplo, enumera siete bienes fundamentales: vida, conocimiento, juego, belleza, amistad, razonabilidad práctica, re-

9 Cfr. en particular B. Celano, Derecho, justicia, razones, op. cit., 23-60.

10 Así, M. WEBER, «Wissenschaft als Beruf» (1919), trad. cast. «La ciencia como profesión» en id., La política como profesión, Madrid, Espasa Calpe, 1992. C. SCHMITT, Politische Theologie (1922), trad. cast. Teología política, Madrid, Trotta, 2009, extiende la clasificación a valores económicos (útiles/desventajosos) y políticos (amigo/enemigo).

${ }_{11}$ Este es el núcleo de la acusación de metafísica dirigido por el neopositivismo lógico a las filosofías tradicionales. Sucesivamente, muchas distinciones neopositivistas se han debilitado, pero tomarlas en serio, aunque solo sea para criticarlas, sigue siendo quizá el único aspecto distintivo de una filosofía o teoría analítica. 
ligión, pero no puede, sin embargo, calificársele como pluralista porque no contempla la posibilidad de conflicto entre ellos ${ }^{12}$. Para el pluralismo, por su parte, los valores son conflictivos, pero sus conflictos son conciliables. Es siempre posible llegar a trade-offs, compromisos o balances que, sin embargo, pueden exigir elecciones trágicas; esta es la idea distintiva del pluralismo ${ }^{13}$.

Como ya notaba Max WeBER, que se den desacuerdos éticos o prácticos, más o menos extensos según las épocas y las culturas, es un hecho empírico importante pero no decisivo para la alternativa objetivismo/subjetivismo ${ }^{14}$. Igualmente decisivo es cómo los desacuerdos son definidos; por ejemplo, ¿sólo prima facie o también all things considered? Finalmente, no menos importante es la dimensión normativa o valorativa. En la ética antigua, típica de sociedades face to face y cerradas hacia el exterior, la armonía era un valor casi indiscutido. En la ética moderna, en cambio, se vuelve sostenible la idea maquiavélica de la productividad del conflicto.

Sobre estos temas la literatura pluralista adopta una actitud realista, en el sentido del realismo político y jurídico. Este resulta ser un buen punto de partida para afrontar problemas como el conflicto entre libertad y seguridad. A diferencia del relativismo (meta) ético para el cual, después de todo, anything goes, el pluralismo toma en serio el conflicto, salvo hacer poco más que admitirlo y plantear compromisos. El nuevo constitucionalismo, en cambio, es menos drástico sobre los conflictos pero más interesado en su solución para lo cual adopta técnicas como el balance y el test de proporcionalidad (cfr. 3.3).

\section{4. ¿Valores éticos inconmensurables?}

«Inconmensurabilidad» admite dos sentidos principales. En el primer sentido, fuerte, indica imposibilidad de comparación, alteridad o heterogeneidad entre valores ${ }^{15}$. En este sentido, a veces, entre valores inconmensurables se postulan elecciones radicales, es decir, absolutas (sin que puedan ser contemperadas) y sin razones (que no sean ficticias). Piénsese en la elección entre verdad y ética propuesta por WEBER para científicos y docentes universitarios, y que solo depende, en última instancia, de la vocación (Beruf) del docente. Por otro lado, también elecciones como estas bien pueden ser racionales en el doble sentido: de no ser absolutas pero, en todo caso, de admitir razones ${ }^{16}$.

En el segundo sentido, débil, «inconmensurabilidad» solo significa ausencia de una medida común y de un orden de preferencia prefijado. Los monistas a menudo han

12 Cfr. J. FinNIS, Natural Law and Natural Rights (1980: 1992), trad. it. Legge naturale e diritti naturali. Torino, Giappichelli 1996, 93-105.

13 Cfr., paradigmáticamente, I. BERLIN, «My Intellectual Path», op. cit., y B. Williams, «Liberalism and Loss», en M. LILA, R. Dworkin, y R. B. Silvers (eds.), The Legacy of Isaiah Berlin, New York, New York Review of Books, 91-104.

${ }^{14}$ Cfr. M. WeBER, «Der Sinn der "Wertfreiheit" der soziologischen und ökonomischen Wissenschaften» (1917), trad. it. en id, Il metodo delle scienze storico-sociali, Milán, Mondadori, 1958, 326: «Sobre estas cuestiones no se llega a nada a través de constataciones de hecho».

${ }_{15}$ Cfr., al menos, R. ChAng (ed.), Incommensurability, Incomparability and Practical Reason, Cambridge (Mass.), Harvard U. P., 1997.

${ }_{16}$ La alternativa elección radical/racional y otras alternativas solo aparentemente dicotómicas, es propuesta por J. GRAY, Isaiah Berlin (1995), trad. cast. Isaiab Berlin, Valencia, Alfons el Magnànim, 1996, e id., Two Faces of Liberalism (2000), trad. cast. Las dos caras del liberalismo, Barcelona, Paidós, 2001. 
admitido una suerte de medida común a todos los valores (la utilidad) o a todos los derechos (la dignidad humana), que proveería también el criterio para ordenarlos $e x$ ante, es decir, para proveer una jerarquía axiológica preestablecida apta para solucionar los (aparentes) conflictos. Para los pluralistas, en cambio, una medida y un orden semejante no se dan a priori, pero pueden buscarse solo a posteriori, realizando tradeoffs, compromisos o balances, todas ellas, obviamente, actividades ni irracionales ni, menos aún, irrazonables.

La inconmensurabilidad, en ambos sentidos, es un problema teóricamente insoluble que admite solo soluciones prácticas. En los casos de inconmensurabilidad, fuerte o débil, alguien debe finalmente decidir, radicalmente o racionalmente. Este problema es dejado abierto por el pluralismo de los valores y afrontado por el derecho del nuevo constitucionalismo. Este instituye autoridades normativas que conmensuran valores inconmensurables en los dos sentidos, como libertad y seguridad; el nuevo constitucionalismo, en particular, confía el control de sus decisiones a cortes supremas, constitucionales e internacionales (cfr. 3.4).

\section{5. ¿Valores éticos indeterminados?}

Finalmente, monistas y pluralistas pueden concordar sobre la objetividad de los valores, pero discrepan sobre su determinación. Los valores son objetivos sea para los monistas como para los pluralistas; para los primeros, porque son natural o racionalmente dados; para los segundos, porque son alcanzados mediante abstracciones de juicios de valor formulados en las distintas culturas. En cuanto a su determinación, en cambio, para los monistas son reducibles a la unidad, armónicos, conmensurables y, en conjunto, determinados, mientras que para los pluralistas siempre quedan múltiples, conflictivos, inconmensurables y, en conjunto, indeterminados.

Determinación e indeterminación pueden ser entendidos como un rasgo en sí mismo: la genericidad ( $c f r$. 3.5), o como el conjunto de los rasgos anteriores, a lo que, sin embargo, hace falta añadir algo. Las autoridades jurídicas llamadas a elegir, balancear y conmensurar valores realizan determinaciones que quedan, en alguna medida, fatalmente subjetivas, particulares y contextuales. Cada uno de estos adjetivos, usados de forma distinta en filosofía moral y en teoría del derecho, se presta a equívocos; por tanto, es necesario precisar que aquí solo son relevantes los sentidos jurídicos, sobre los que se puede decir cuanto sigue.

Las determinaciones de las autoridades jurídicas son subjetivas, antes que nada, en el sentido de discrecionales: elecciones entre posibles alternativas. Luego, son particularistas, pero no en el sentido del particularismo moral (relativo a casos concretos) sino en el sentido jurídico de ser estructuralmente inestables, siempre pasibles de excepciones en casos posteriores. Finalmente, tales determinaciones son contextuales precisamente en el sentido de ser relativas a diversos contextos que, sin embargo, pueden ser más o menos abstractos en las determinaciones legislativas, o bien concretos, pero solo en las determinaciones judiciales ordinarias ${ }^{17}$.

17 Para todas estas precisiones $c f r$ : en términos de «no trascendibilidad del poder decisorio», B. CELANO, «Rule of Law y particularismo ético», en P. LuQue (ed.), Particularismo. Ensayos de filosofía del derecho y 


\section{NUEVO CONSTITUCIONALISMO}

En Occidente, debido a la prioridad cronológica del ius romano sobre el cristianis$\mathrm{mo}^{18}$, el derecho ha jugado un rol central que en otros lugares ha cumplido la religión o la costumbre. En particular, la determinación de los valores ha sido confiada, inicialmente, a una clase de juristas (Estado jurisdiccional), luego, tras la Revolución francesa, al legislador democrático (Estado legislativo), finalmente, después de Auschwitz, a constituyentes, legisladores y jueces, sean ordinarios o constitucionales (Estado constitucional). «Nuevo constitucionalismo» designa precisamente a las instituciones de esta última forma de Estado, instituciones a no confundir con el neoconstitucionalismo, de las que representa su teoría ${ }^{19}$.

Después de la Segunda Guerra Mundial, con base en el modelo de la Constitución federal estadounidense, han empezado a difundirse las sucesivas instituciones constitucionales. Ante todo, constituciones rígidas y más o menos «extensas», con declaraciones de los derechos que a menudo se extienden a derechos sociales y de otros tipos. Luego, distintas formas de control de constitucionalidad: judicial review, control concentrado, recurso directo o amparo. Finalmente, por efecto de tales instituciones, el derecho se ha «constitucionalizado»: por medio de la interpretación constitucional, los principios se han «irradiado» a todo el cuerpo del derecho ${ }^{20}$.

Se podría dudar que el new constitutionalism posbélico representa una novedad absoluta. Rafa ESCUDERO, por ejemplo, ha destacado importantes precedentes prebélicos ${ }^{21}$. No obstante, dos aspectos pueden considerarse nuevos: la aceptación, después de Auschwitz, de la tradición liberal de los derechos por parte de las tradiciones cristiana y socialista, hasta entonces hostiles y la transformación de las declaraciones de los derechos en documentos jurídicos, aplicables por jueces constitucionales u ordinarios $^{22}$. Ciertamente, se trata de documentos jurídicos bien extraños, que en lugar de determinar los valores éticos en reglas, parecen limitarse a reformularlos en principios igualmente indeterminados.

En efecto, si solo fuese por las declaraciones de los derechos, el nuevo constitucionalismo solo parecería un tropiezo en el proceso de determinación de los valores éticos en reglas jurídicas. Sin embargo, los principios del nuevo constitucionalismo son determinados por las Cortes supremas, constitucionales e internacionales, a través de la denominada interpretación constitucional: técnicas como el balance (Abwägung

filosofía moral, Madrid, Marcial Pons, 2015, especialmente 167-176; en términos de «premisa institucional», T. ENDYCOTT, «Proportionality and Incommensurability», Legal Research Paper Series n. 40/2012, University of Oxford, en ssrn.com/abstract=2086622, 17.

${ }_{18}$ Cfr. M. BARBERIS, «Libertà religiosa», en Ragion pratica, 40/1, 2013, 175-189.

${ }_{19}$ Cfr., al menos, P. ComanducCI, Constitución y teoría del derecho, México, Fontamara, 2007, y M. BARBERIS, Stato costituzionale. Sul nuovo costituzionalismo, Módena, Mucchi, 2013.

${ }_{20}$ «Constitucionalización» e «irradiación» son expresiones usadas, respectivamente, por Riccardo GuASTINI y Robert ALEXY.

${ }_{21}$ Cfr. R. EsCUDERo, Las huellas del neoconstitucionalismo. Democracia, participación y justicia social en la Constitución española de 1931, inédito.

${ }_{22}$ Cfr. R. CubEDDU, «Dai diritti naturali ai diritti umani», en F. SCIACCA (coord.), La libertà in Occidente, Soveria Mannelli (Cz), Rubbettino, 2011, 9-39 y muchos trabajos de Michel TrOPER, a partir de los ensayos recogidos en id., La théorie du droit, le droit, l'État, Paris, Puf, 2001. 
balancing) y los tests de proporcionalidad (3.4 y 4.3.3). Por otra parte, de tales principios constitucionales resultan predicables las mismas características que el pluralismo atribuye a los valores: objetividad, pluralidad, conflictividad, inconmensurabilidad, indeterminación.

\section{1. ¿Principios constitucionales objetivos?}

Las constituciones y declaraciones de los derechos posbélicos se presentan como tablas de los valores; piénsese solo en el Tratado de Niza (2001) y en su sistemática, denominada precisamente «por valores». La jurisprudencia constitucional usa tales documentos como un menú tendencialmente completo del que extraer principios para cada caso que pueda presentarse; principios tanto explícitos, es decir, expresamente formulados en el documento, pero también implícitos, reconstruidos por los propios intérpretes. A unos u otros, según qué prevalezca o no en el balance, se atribuye luego carácter fundamental, supremo o último ${ }^{23}$.

En la famosa sentencia LUTH (1958) del Tribunal constitucional alemán, este menú de principios es caracterizado como un «orden objetivo de valores», pero «orden» y «objetivo» ¿en qué sentido? Los principios forman un orden en tanto que indivisibles, como en el Preámbulo del Tratado: «La Unión está fundada sobre los valores indivisibles y universales de la dignidad humana, la libertad, la igualdad y la solidaridad». Es decir, que gran parte de las jurisprudencias constitucionales cree que los principios, lejos del constituir un mero conjunto identificado extensionalmente por sus componentes, forman un sistema identificado intensionalmente por las relaciones entre tales componentes ${ }^{24}$.

Dice por ejemplo la Corte constitucional italiana en la decisión 85/2013, sobre el denominado caso Ilva, reenviando a la anterior sentencia 264/2012: «Todos los derechos fundamentales tutelados por la Constitución se encuentran en relación de integración recíproca [...] La tutela debe ser siempre "sistémica y no fraccionada en una serie de normas no coordinadas y en potencial conflicto entre ellas"». Dicho de otro modo, el nuevo constitucionalismo parece mediar entre la retórica monista y la retórica pluralista recogiendo la tradicional idea jurídica de sistema: los posibles conflictos entre principios constitucionales se remedian mediante una interpretación sistemática.

Así, el orden de los principios es objetivo por una razón localizable, en su forma más clara, en Robert ALEXY, máximo apologeta del new costitutionalism y el mayor teórico neoconstitucionalista. Los valores se vuelven objetivos en tanto que extraídos de juicios de valor subjetivos mediante procesos de abstracción que los hacen cada vez más indeterminados ${ }^{25}$ ( $c f r$. 2.1). Como para los valores, según CELANO, pero en un

${ }^{23}$ Cfr. P. Faraguna, A. Ai confini della costituzione. Principi supremi e identità costituzionale, Milán, Angeli, 2015.

${ }_{24}$ Cfr. www.cortecostituzionale.it. A favor de una perspectiva sistemática, cfr. M. CARTABIA, I principi di ragionevolezza e proporzionalità nella giurisprudenza costituzionale italiana, en www.cortecostituzionale.it/ documenti/convegni/seminari/RI_Cartabia_Roma2013.pdf, en particular, 9-10; en contra, R. GUASTINI, «I limiti intrinseci alla revisione costituzionale nella giurisprudenza colombiana», inédito, 19-20, n. 43.

${ }_{25}$ Así R. Alexy, Theorie der Grundrechte (1994), trad. it. Teoria dei diritti fondamentali, Bologna, Il Mulino, 2012, 555-559. 
proceso que se desarrolla también desde lo bajo, y no solo desde lo alto, el máximo de objetividad de los principios constitucionales se obtiene al precio de la máxima indeterminación.

\section{2. ¿Principios constitucionales plurales?}

Si solo se atendiese a los documentos constitucionales, las declaraciones de los derechos del nuevo constitucionalismo podrían parecer monistas o pluralistas. Piénsese aún en el Tratado de Niza, que inicia con un principio fundamental, la dignidad humana, pero luego adopta una sistemática «por valores» que pone a todos los principios sobre el mismo plano. En realidad, el tenor literal del texto es menos importante que su interpretación, a propósito de la cual se opone, a veces, una perspectiva dignitarian europea y latino-americana, más monista y holista, y una libertarian norteamericana, más pluralista e individualista ${ }^{26}$.

En realidad, estas dos perspectivas solo parecen diversos mix de aplicación deductiva (categorical approach), privilegiada por los justices norteamericanos, y de balance, preferido por los jueces continentales ${ }^{27}$. Como ya hemos señalado, en la retórica del new constitutionalism se combina monismo y pluralismo, coherentismo y conflictualismo. Leemos aún la decisión 85/2013 de la Corte constitucional italiana, que en pocas líneas reformula muchos de estos elementos en términos de cuatro fórmulas mágicas del nuevo constitucionalismo: balance, proporcionalidad, razonabilidad y núcleo esencial de los derechos.

La Constitución italiana, como las otras Constituciones democráticas y pluralistas contemporáneas, exige un continuo y recíproco balance entre principios y derechos fundamentales, sin pretensiones de absolutismo por ninguno de ellos [...]. El punto de equilibrio, precisamente porque dinámico y no prefijado por anticipado, debe ser valorado por el legislador en el establecimiento de las normas, y por el juez de las leyes en el ámbito del control, según criterios de proporcionalidad y razonabilidad, tales de no permitir un sacrificio de su núcleo esencial.

\section{3. ¿Principios constitucionales conflictivos?}

Los juristas han estado siempre esquizofrénicamente divididos entre una teoría coherentista del sistema jurídico y una práctica conflictualista de la solución de las antinomias. Prima facie, el derecho, el conjunto de los materiales jurídicos, es antinómico; all things considered, después de su sistematización por parte de los juristas, se vuelve un sistema jurídico más o menos coherente ${ }^{28}$. En el caso de los principios constitucio-

26 Así, M. A. GLENDON, «Rights Babel. Thoughts on Approaching 50th Anniversary of the Universal Declaration of Human Rights», en Gregorianum, 79, 4, 1998, 611-624; cfr. también C. M. MCCRUDDEN, «Human Dignity and Judicial Interpretation of Human Rights», en The European Journal of International Law, 19, 4, 2008, 655-724.

${ }_{27}$ Cfr. M. Rosenfeld, «Constitutional Adjudication in Europe and United States: Paradoxes and Contrasts», en $I \bullet$ Con, 2004, 2/4, 633-668. 2014.

${ }_{28}$ Me permito reenviar al capítulo cuarto de mi Introduzione allo studio del diritto, Torino, Giappichelli, 
nales, la posibilidad de conflictos aumenta exponencialmente con el alargamiento de las constituciones y la inflación de los derechos; más principios se suman al menú, más conflictos son posibles.

Las constituciones del nuevo constitucionalismo tienden a la plenitud, pretenden abarcar, explícita o implícitamente, todos los valores presentes en sociedades pluralistas. Plenitud y coherencia, sin embargo, son a su vez ideales conflictivos, la búsqueda de uno ocurre en detrimento del otro. El balance, aunque sea la solución estándar del conflicto entre principios, no inmuniza ciertamente de la incoherencia, pero puede reducirla al máximo. Al respecto, Alexy distingue dos modelos del balance: uno crítico, denominado de la decisión (Dezisionmodell), y uno apologético, denominado de la justificación (Begrundungsmodell) ${ }^{29}$.

Se trata de modelos también en el sentido que, de un lado, un mismo balance puede ser representado de ambos modos; del otro, distintos balances, realizados por diferentes cortes, pueden corresponder más a uno o al otro ( $c f r$. 4.3.3.2). Aquí, en seguida, indico brevemente tres puntos discutidos por los defensores de los dos modelos, mostrando cómo el modelo de la justificación es más explicativo que el modelo de la decisión. Los puntos controvertidos son relativos al hecho que el balance: 1) sea una elección a secas o bien una acomodación; 2) esté referido a casos concretos o más o menos abstractos; 3 ) determina jerarquías móviles o bien fijas.

3.3.1. En cuanto a la elección a secas o una acomodación, muchas sentencias parecen realizar una elección a secas a partir de un único valor-principio: piénsese en la aplicación del principio de igualdad hecha por la Corte Suprema estadounidense en Brown vs. Board of Education (1954). Otras veces, en decisiones sobre el aborto, como Roe vs. Wade (1973) de la misma Corte Suprema, y como la sentencia 27/1975 de la Corte constitucional italiana, se declara más o menos expresamente acomodar principios conflictivos: en el primer caso, el interés estatal a la salud y el derecho a la privacy de la madre; en el segundo, el derecho a la vida del concebido y el derecho a la salud de la mujer ${ }^{30}$.

3.3.2. En cuanto a decisiones en abstracto y en concreto, las Cortes siempre deciden, por definición, caso por caso; sin embargo, por el control de constitucionalidad, «caso» puede significar caso concreto o bien más o menos abstracto, según el tipo de control. En el amparo, o recurso directo, el caso es concreto; en el control de constitucionalidad concentrado es abstracto; en el judicial review puede ser concreto o bien más o menos abstracto. Por otro lado, también un caso concreto es tal caso en tanto que identificado en términos abstractos ${ }^{31}$; en todo caso, también de una decisión concreta se pueden abstraer reglas más o menos abstractas o principios más o menos genéricos

29 Cfr. R. AleXY, Theorie der Grundrechte, trad. it. cit., 182-197. Según M. COHEN-EliYa, I. PoRAT, Proportionality and Constitutional Culture, Cambridge, Cambridge U.P., 2013, el nuevo constitucionalismo global reemplazaría la cultura de la autoridad por una cultura de la justificación.

${ }_{30}$ Para una crítica a la formulación de Roe, $c f r$. G. CALABRESI, Il mestiere di giudice. Pensieri di un accademico americano, Bologna, Il Mulino, 2013, 62-64.

31 Una conducta concreta constituye homicidio, corrupción, ruptura de una promesa solo en relación con los conceptos abstractos correspondientes: $c f r$. B. CELANO, «True Exceptions: Defeasibility and Particularism», en J. Ferrer Beltrán y G. B. RAtTi (eds.), The Logic of Legal Requirements, Oxford, Oxford U. P., 2012, 280, n. 36. 
3.3.3. En cuanto a jerarquías móviles o fijas, las decisiones judiciales uti singulae producen jerarquías móviles, variables sea de caso concreto a caso concreto, sea de caso abstracto a caso abstracto. Uti universae, en cambio, las decisiones producen jerarquías ciertamente no fijas pero, a menudo, relativamente estables. Parece admitirlo también Riccardo GuASTINI, principal partidario de la movilidad de las jerarquías, cuando afirma que en el balance no prevalecen los principios supremos sino que se vuelven supremos los principios que prevalecen ${ }^{32}$. En efecto, la solución de todos los posibles conflictos no podría ser prevista por el constituyente ex ante, solo puede ser establecida por los jueces ex post.

\section{4. ¿Principios jurídicos inconmensurables?}

En un famoso ensayo, el primer partidario del modelo de la decisión, Carl SCHMITT, acusó al Tribunal constitucional alemán de querer «hacer conmensurable lo inconmensurable» ${ }^{33}$. Pero esto es cuanto han hecho siempre los jueces ordinarios, mucho antes que los jueces constitucionales, y en materias paradigmáticamente jurídicas como la responsabilidad civil y penal. Los jueces siempre han sido llamados a conmensurar valores inconmensurables, tanto en el sentido fuerte de incomparables como en el sentido débil de carentes de una medida común ( $c f r$. 2.4), como la vida, el honor, la propiedad ${ }^{34}$. El problema no es, entonces, la conmensuración sino los criterios con base en los que sucede.

Si los valores son inconmensurables, entonces no pueden ser conmensurados simplemente cuantificándolos como proponen los utilitaristas en filosofía moral pero también teóricos del balance como ALEXY. Después de todo, balanza, ponderación y peso de los principios son solo metáforas, aplicadas a bienes que no pueden, literalmente, pesarse, medirse y balancearse. De los propios test de legitimidad, idoneidad, necesidad, proporcionalidad en sentido estricto, empleado por los jueces continentales para expresar los pasos del balance ${ }^{35}$, veremos que solo el segundo — la idoneidad- se presta a valoraciones objetivas, de racionalidad instrumental, medio-fin.

El balance de las razones en pro y en contra de una cierta conducta de acción es considerado hoy la forma general del razonamiento práctico, respecto al cual el razonamiento jurídico, norm- o rule-based, solo constituye el caso particular ${ }^{36}$. El balance

32 Cfr. en particular R. GUASTINI, «Gerarchie normative», Materiali per una storia della cultura giuridica, 1997, 463-487, e id., Teoria e dogmatica delle fonti, Milán, Giuffrè, 1998, 124-125, además, para una discusión del punto, J. FERRER BELTRÁN y J. L. RODRÍGUEZ, Jerarquías normativas y dinámica de los sistemas jurídicos, Madrid, Marcial Pons, 2011, 143-146.

33 Así, C. Schmitt, Die Tyrannei der Werte (1967), trad. it. La tirannia dei valori, Roma, Pellicani, 1987, 30. Cabe recordar que el denominado decisionismo schmittiano deriva de una radicalización de las tesis antiformalistas sostenidas en su juventud Gesetz und Urteil (1912).

34 Lo recuerda T. ENDYCOTT, Proportionality and Incommensurability, op. cit., 14.

35 Así, M. COHen Elyia y I. PORAT, Proportionality and Constitutional Culture, op. cit., 16-23. Sobre el tema, cfr. también A. BARAK, Proportionality, Constitutional Rights and their Limitations, Cambridge, Cambridge U. P., 2012.

36 Así, según el modelo a dos niveles del razonamiento práctico, propuesto por J. RAZ, Practical Reason and Norms (1979), Oxford, Oxford U. P., 1990, retomado por F. SCHAUER, Playing by the Rules, Oxford, Clarendon, 1991 y también acogido por M. AtIEnZA, Curso de argumentación jurídica, Madrid, Trotta, 2013. 
de los principios, típico del nuevo constitucionalismo, representa un caso particular del caso particular o, si se quiere, un retorno al caso general. En el caso de los balances libertad-seguridad, es el ejecutivo el llamado a tomar medidas de emergencia, bajo el control inmediato del legislativo, y el sucesivo control judicial.

Aquí se puede hablar de balance en otro sentido, no deliberativo sino institucional, en el sentido de los checks and balances entre poderes. En abstracto, hay tres modelos de gestión de la emergencia: uno basado en los plenos poderes del ejecutivo, el otro en el respeto absoluto de las libertades constitucionales y el tercero, precisamente, en los checks and balances entre los tres poderes del Estado ${ }^{37}$. El nuevo constitucionalismo adopta el tercer modelo, en el que puede tocarles a los jueces controlar los balances libertad-seguridad realizado por los otros poderes. Es aquí, como veremos en las secciones conclusivas (apartados 4 y 5), que se replantean los problemas de inconmensurabilidad entre valores-principios en juego.

\section{5. ¿Principios jurídicos indeterminados?}

La indeterminación de los principios, como la de los valores, podría entenderse como el conjunto de los rasgos considerados hasta ahora, o bien como un rasgo ulterior: la genericidad ${ }^{38}$. Esta es, a menudo, confundida con la vaguedad, es decir, con el halo de incertidumbre que circunda la denotación de cada término, haciendo dudoso si incluye o no a un objeto. En los significados genéricos, en cambio, no surgen dudas de este tipo: principios genéricos, formulados en términos de justicia, dignidad, utilidad, seguridad, pueden aplicarse virtualmente a cualquier caso. Un sistema constitucional formulado «por principios» puede, pues, aspirar realmente a la plenitud.

El problema es que, mientras que un caso solo esté regulado genéricamente, no sabemos cómo está regulado; para saberlo, hace falta especificar los principios en reglas. Para cumplir con su función, determinar los valores éticos, el derecho debe estar formado sobre todo por reglas, no solo por principios; una declaración de derechos humanos, en sí misma, es aún un documento moral. Sin embargo, como ha mostrado Cass Sunstein, en la formulación de constituciones para sociedades pluralistas, los denominados acuerdos no teorizados pueden alcanzarse en dos modos, que muestran cómo las reglas y los principios pueden ser usados para el mismo objetivo: maximizar el acuerdo y minimizar el desacuerdo ${ }^{39}$.

A veces los constituyentes encuentran el acuerdo sobre una regla abstracta, de la que deducir decisiones judiciales concretas, dejando indeterminado el principio que la justifica, sobre el que podría haber desacuerdo. Más a menudo, en cambio, los constituyentes encuentran el acuerdo sobre un principio genérico, dejando así a los legisladores la especificación de reglas abstractas, y a los gobernantes y a los jueces la

37 Cfr. M. Rosenfeld, «Judicial Balancing in Times of Stress: Comparing Different Approaches to the War on Terror», Cardozo Legal Studies Research Paper núm. 119, 1 de abril de 2006, en: ssrn.com/abstract $=729224,4$.

38 Cfr. al menos C. LuZZATI, Príncipi e principi. La genericità nel diritto, Torino, Giappichelli, 2013.

39 Cfr. C. Sunstein, Designing Democracy, Oxford, Oxford U. P., 2001, 49-66, reformulado aquí en términos de reglas abstractas y principios genéricos. 
toma de decisiones concretas. Como vemos, pasando finalmente a las relaciones entre libertad y seguridad, estas no son dejadas solo al balance de los principios, hay también auténticas reglas como la prohibición de la tortura.

\section{SEGURIDAD Y LIBERTAD}

Las reacciones a los ataques terroristas del 11 de septiembre de 2001 en los Estados Unidos, y del 13 de noviembre de 2015 en París, siguen un mismo guión, en gran parte ya escrito en el siglo $\mathrm{XX}$, siglo en el que dos guerras mundiales y varios tipos de terrorismo han desplazado hacia los ejecutivos poderes sin precedentes. Que los gobiernos sean de derecha o de izquierda, que se declare o no el estado de emergencia, gobernantes en ausencia de consenso se descubren como commanders in chief y proclaman la «guerra al terror» (wars on terror) o asimétricas o híbridas, una suerte de tertium quid entre la auténtica guerra y la simple represión del terrorismo.

Si se tratase de una auténtica guerra, a los prisioneros de guerra se les aplicarían las garantías previstas en la Convención de Ginebra (1949). Si se tratase de simple terrorismo, a los terroristas se les aplicarían las garantías previstas en el derecho penal interno. La guerra al terror, en cambio, permite adoptar medidas extrañas al derecho, tanto internacional como interno: detenciones sin acusaciones y sin proceso de sospechosos calificados como enemy combatants, eventualmente también en prisiones secretas (black holes) en el extranjero, homicidios dirigidos (targeted killings) o extraordinary renditions de los sospechosos hacia países que practican la tortura.

Si solo se tratase de medidas excepcionales, todo ello se reconduciría a la casuística de los dilemas morales y las elecciones trágicas admitida por el pluralismo de los valores. En realidad, sobre una sola cosa concuerdan los liberales, defensores del Estado constitucional y de las libertades individuales, y los abogados de la emergencia, que hablan del derecho penal del enemigo (Feindstraftrecht) y de legalizar la tortura ${ }^{40}$. El terrorismo global y la guerra al terror amenazan con transformarse de situaciones excepcionales a escenarios permanentes, con el ulterior riesgo de transformar progresivamente el Estado constitucional, nacido de la victoria sobre los totalitarismos, en Estado de seguridad.

En este contexto, la metáfora de los balances entre libertad y seguridad, es a menudo un simple artificio retórico al servicio de la propaganda de los ejecutivos ${ }^{41}$. Sin embargo, usar este mismo vocabulario, en los modos más rigurosos sugeridos por el pluralismo de los valores y el nuevo constitucionalismo, permite ver con mayor claridad las alternativas efectivas, y hace posible plantear el cuadro constitucional dentro

40 Entre los primeros, cfr., al menos, B. ACKERMAn, «The Emergency Constitution», en Yale Law Journal, 113, 2004, 1029-1091 y M. ROSENFELD, «Is Judicial Balancing Appropriate in the War of Terror? Contrasting Ordinary Times, Emergencies, and Times of Stress», en Percorsi costituzionali, 1, 2008 (número monográfico completamente dedicado a «Libertad y seguridad»), 139-148. Entre los segundos, además de Günther JAKOBS, que ha teorizado el Feindstraftrecht en varias ponencias de congresos, cfr. A. DeRSHOwITZ, Why Terrorism Works. Understanding the Threat, responding to the Challenge, New Haven (Ct), Yale U. P., 2004.

${ }^{41}$ Así D. BIgO, R. B. J. WALKER et al. ELISE, «Liberté et securité en Europas: enjeux contemporains», en Culture et conflits, 61, 2006; D. BigO et al. (eds.), Europe's 21st Century Challenge: Delivering Liberty, Farnham (Surr), Ashgate, 2010. 
del que debe desarrollarse la política de la emergencia. Seguidamente se examinan, en particular, las derivaciones institucionales producidas por la guerra al terror (4.1) y las posibles respuestas, solo políticas (4.2) o también jurídicas (4.3) a tales problemas.

\subsection{Derivaciones institucionales}

La principal consecuencia institucional de la guerra al terror es la ulterior consolidación de los ejecutivos a costa del legislativo y del judicial, siguiendo así un proceso en curso de al menos un siglo, pero que hoy amenaza transformar el Estado constitucional en Estado de seguridad ${ }^{42}$. El resultado que no han producido siquiera dos guerras mundiales, concentrando en los gobiernos poderes inauditos, podría producirlo la war on terror: vaciar definitivamente los poderes del Parlamento y hacer vanos los remedios judiciales. Como profetizó Walter BENJAMín, a propósito de la república de WEIMAR, el estado de excepción puede volverse permanente ${ }^{43}$.

No se reflexiona lo suficiente sobre cuánto hayan cambiado dos guerras mundiales los equilibrios entre los poderes del Estado, con respecto al modelo de la separación de los poderes de MonTESQuieu. La Constitución federal de los Estados Unidos, el arquetipo del nuevo constitucionalismo, puso en equilibrio los poderes legislativo, ejecutivo y judicial; fueron dos guerras mundiales las que inclinaron la balanza a favor del ejecutivo. Pero un ejemplo aún más general es la delegación a los gobiernos de gran parte de la legislación, delegación iniciada, también ella, a título de declaración de urgencia, pero que va transformando las democracias representativas en autocracias electivas ${ }^{44}$.

El proceso de concentración de los poderes en el ejecutivo en razón de la emergencia terrorista interesa a todos los países de Occidente, hayan sido atacados o no. Los Estados Unidos tienen su Patriot Act (2001) y Guantánamo; Francia se propone constitucionalizar el état d'urgence y quitar la ciudadanía a los terroristas, como ha denunciado, al dimitir, la ministra de justicia Christiane Taubira; el Reino Unido se ha dado leyes antiterrorismo draconianas; incluso Italia se ha dado la ley Bossi-Fini sobre la inmigración (2002). Todas medidas propuestas por los gobiernos, aprobadas sin discusión por los Parlamentos, y solo parcialmente corregidas por los jueces.

El Report 2016 de Human Rights Watch denuncia el vaciado de los derechos humanos y del rule of law cumplido por esta «política del miedo» ${ }^{45}$. Contra estas derivaciones, sin embargo, los partidarios de los derechos humanos solo pueden emplear dos estrategias institucionales muy débiles, justamente porque basadas sobre los poderes legislativo y judicial, uno controlado por el ejecutivo, el otro deferente hacia el ejecuti-

42 Expresiones como «Security State» y «État de securité» se encuentran solo en quien denuncia el fenómeno: cfr., desde los títulos, M. G. RASKIN y R. SPERO, The Four Freedoms under Siege: the Clear and Present Danger from Our National Security State, Westport, Praeger, 2007 y G. Agamben, De l'État de droit à l'État de Securité, en www.lemonde.fr, 27 de diciembre de 2015.

${ }^{43}$ Cfr. G. Agamben, Stato di eccezione, Torino, Bollati Boringhieri, 2004.

${ }^{44} \mathrm{La}$ expresión es de M. BOVERO, Seguridad jurídica y democracia. Un perspectiva teórico-política, en C. Cruz Moratones, C. Fernández Blanco y J. Ferrer Beltrán (eds.), Seguridad jurídica y democracia en Iberoamérica, Madrid, Marcial Pons, 2015, 49-57.

45 Human Rights Watch, Worldreport 2016, Nueva York, Seven Stories, 2016. 
vo y el legislativo. Una de las dos respuestas es sobre todo política, orientada a cambiar la opinión pública, los Parlamentos y los gobiernos; la otra, es predominantemente jurídica en cuanto apela sobre todo a las cortes, internas e internacionales.

\subsection{Una respuesta (solo) política}

El mayor representante de la respuesta política a las derivaciones en seguridad es el Jeremy WALDRON de Torture, Terror and Trade-offs (2010), colección de ensayos sobre las reacciones al 11 de septiembre. Waldron es el más conocido crítico del judicial review; su respuesta, por tanto, es meramente política y no judicial. No obstante, estos ensayos proveen un análisis de la seguridad y cinco argumentos contra el sacrificio de la libertad en su nombre, que deben ser considerados sea por sus méritos, sea porque resultan pertinentes en una perspectiva jurídica. En esta última perspectiva, sobre todo el primer argumento juega un papel decisivo: la no independencia de libertad y seguridad.

\subsubsection{No-independencia de libertad y seguridad}

En la teoría de la justicia angloamericana y en el derecho público europeo-continental, seguridad y libertad son opuestas como valores inconmensurables. La primera, es el bien público por antonomasia; la segunda, el derecho individual par excellence. También por esto sus relaciones son representadas en términos de balanza como un juego de suma cero: más seguridad igual menor libertad, y viceversa. Sin embargo, analizando el concepto de seguridad, como solo lo han hecho Thomas HoBBES, Jeremy BENTHAM y MONTESQUIEU, uno se percata que, lejos del ser incomparables, libertad y seguridad presentan relaciones internas, definitorias o conceptuales.

The idea of a trade-off between liberty and security make clearest sense if we think of liberty and security as separate values, logically independent of one another, and related in a sort of inverse way: the more liberty there is, the less security you are likely to get; the more security you want, the more liberty you're going to have to give up in order to get it. But if we find that liberty and security are not logically independent and that there are important internal relation between them, or if conceptual analysis indicates that they stand sometimes in a direct rather than in an inverse relation to one another, then talk of a trade-off will be complicated, if not undermined ${ }^{46 .}$

WALDRON invita a concebir la seguridad como un derecho individual análogo a la libertad ${ }^{47}$, propuesta menos innovadora de lo que podría parecer. También MONTESQUIEU definió la libertad como la opinión, nutrida por cada uno, acerca de la propia seguridad. Incluso Judith SHKLAR y WILLIAMS, pluralistas amigos de BERLIN, han de-

46 Así J. Waldron, Torture, Terror and Trade-offs. Philosophy for the White House, Oxord, Oxford U. P., 2010, 160-161. Importantes objeciones a WALDRON se encuentran en A. PINTORE, «Tortura, sicurezza e argomenti. A proposito di un libro di Jeremy Waldron», en Diritto e questioni pubbliche, 2010, 10, 579-600.

47 El ensayo original, Security as Basic Right (2009), reeditado en J. WALDRON, Torture, Terror and TradeOffs, op. cit., 166-185. H. SHuE, Basic Rights: Subsistence, Affluence, and U. S. Foreign Policy, 1980, 1996, Princeton, Princeton U. P., 1996. 
fendido un liberalismo del miedo (liberalism of fear): una concepción de la libertad como seguridad individual ante las agresiones ajenas, sea de individuos sea de Estados. La misma Convención europea sobre los derechos y libertades fundamentales (1950) asimila libertad y seguridad en el art. 5, como si se tratara de una hendíadis ${ }^{48}$.

En efecto, sobre estas bases, algunos asimilan los dos conceptos ${ }^{49}$. En realidad, los campos semánticos cubiertos por los dos términos no coinciden pero se sobreponen, y precisamente en su núcleo central, esencial e irrenunciable. La libertad liberal, negativa, y la seguridad individual (safety, sûreté), pueden concebirse como derechos a los mismos bienes: vida, integridad física y psíquica, hábeas corpus, derecho a no ser torturado o, en todo caso, dañado injustamente en la persona y en los bienes. Se trata nada menos que de la primera generación de los rights, por tanto, del caso paradigmático e indiscutible de derechos calificados constitucionales, fundamentales y/o humanos.

Fuera de tal núcleo común, «libertad» denota también la libertad positiva y/o la autonomía individual, mientras que «seguridad» se extiende al principal bien colectivo o público asegurado por el derecho y el Estado (security, sécuritée ${ }^{50}$. Entonces, las relaciones entre los dos conceptos pueden representarse como dos círculos que se intersectan y que se sobreponen en un núcleo común de derechos individuales a la libertad-seguridad, como lo denominaré de ahora en adelante. Sin embargo, para tal núcleo común se mantiene la posibilidad de un conflicto (no inter-rights, sino) intraright, o bien, entre los mismos derechos individuales atribuidos a sujetos diferentes (cfr. 4.2.3, por el argumento de la distribución).

\subsubsection{Anti-consecuencialismo}

En la prospectiva libertarian norteamericana ( $c f r .3 .2)$, los derechos no se ponderan, siendo vínculos absolutos a la voluntad de la mayoría; las teorías liberales de la justicia son formuladas de modo que evitan balances entre derechos individuales e intereses colectivos ${ }^{51}$. En la perspectiva dignitarian continental (cfr. 3.2), en cambio, no hay vínculos absolutos. Existe, sin embargo, una presunción liberal, como la llamaré, en la confrontación del sacrificio de derechos individuales por intereses colectivos. Libertades individuales y seguridad colectiva pueden balancearse, pero el sacrificio de

${ }^{48}$ Cfr. Montesquieu, De l'esprit des lois (1748), Paris, Garnier Flammarion, 1979, vol. I, 294: «La liberté politique, dand un citoyen, st cette tranquillité d'esprit qui provient de l'opinion que chacun a de sa sûreté» (XIVI); J. ShKLAR, The Liberalism of Fear, en N. Rosemblum (ed.), Liberalism and Moral Life, Cambridge (Mass.), Harvard U. P., 1989, 21-38; B. Williams, Liberalism of Fear, trad. it., Il liberalismo della paura, en id., In principio era l'azione. Realismo e moralismo nella teoria politica, Milano, Feltrinelli, 2005, 63-75; sobre el art. 5 («Toute personee a droit à la liberté et à la sûreté»), cfr. M. BARBERIS, Europa del diritto. Sull'identità giuridica europea, Bologna, Il Mulino, 2008, 194-198.

${ }_{49}$ Cfr. Th. F. POwERS, «Can We Be Secure and Free?», en The Public Interest, primavera 2003, 3-24.

${ }^{50}$ Una nominalización de los dos sentidos principales de «seguridad» podría identificarse en francés, entre la sûreté individual mencionada por la Déclaration des droits del homme et du citoyen (1789) y la sécurité colectiva: cfr. M. DeLmAS-MARTY, «Dérive sécuritarire», en Le Monde, 26 de marzo de 2016, Idées, 7. Algo análogo podría decirse también para safety y security en inglés.

51 Por dar solo los dos ejemplos más conocidos: con el fin de evitar balances entre derechos individuales e intereses colectivos, el libertarian Robert NOzICK define los derechos como side constraints, mientras que el liberal John RAWLS instituye un orden lexicográfico entre derecho a la igual libertad y derechos a la redistribución. 
las primeras por las segundas exige un strict scrutiny, es decir, justificaciones particularmente rigurosas (cfr. 4.3.3).

\subsubsection{Distribución}

Se ha dicho ( $c f r$. 4.2.1) que también entre derechos a la libertad-seguridad pueden darse conflictos intra-right atribuidos a diversas categorías de personas: mayorías y minorías, ciudadanos y extranjeros, personas cualquiera y sospechosos de terrorismo. Después de Pearl Harbour (1941), inmigrantes y también ciudadanos estadounidenses de origen japonés fueron internados en campos de concentración sin que el Congreso o la Corte Suprema siquiera parpadeen. Las mayorías están siempre dispuestas a sacrificar por la propia seguridad los derechos individuales de las minorías, como si ello no les concerniese. Pero si la libertad es un derecho, es un derecho humano, válido igualmente para todos los seres humanos.

\subsubsection{Efectos no intencionales}

La tutela de la seguridad es considerada, al menos a partir de HOBBES, la función principal del Estado y del derecho. Más aún, la seguridad colectiva constituye una auténtica pre-condición para la tutela de los derechos individuales: sin Estado y sin derecho, estas estarían privadas de tutela. Sin embargo, instituciones planeadas para garantizar la libertad-seguridad individual, siempre pueden ser usadas contra ella. En tales casos, el argumento de la seguridad colectiva como pre-condición de las libertades individuales suena a pretexto, es decir, es legítimo limitar las libertades individuales pero solo si, de otro modo, realmente hubiera riesgo de disolución del Estado ${ }^{52}$ (cfr. 4.3.2-3).

\subsubsection{Consecuencias simbólicas}

La seguridad colectiva es una cuestión de grado. Admitiendo que la restricción de libertades individuales aumenta la seguridad colectiva, lo hace en un porcentaje infinitesimal (¿del 51 al 52 por 100 ?) $)^{53}$ tal que se revela como una medida poco más que simbólica, es decir, para alentar a los ciudadanos y aumentar su confianza en el gobierno, pero ciertamente incapaz de disuadir a terroristas dispuestos al martirio. En el mundo de la comunicación, los símbolos son importantes; para líderes políticos débiles, son decisivos. Pero con base en parámetros más rigurosos como los tests de proporcionalidad, medidas de este tipo aparecen inadecuadas para conseguir los resultados que se proponen ( $c f r$. 4.3.3).

Todos los argumentos de WALDRON, y en particular él último son muy persuasivos pero presentan dos límites estructurales. De momento, son eficaces solo hasta el

52 Cfr. Todo el ensayo Security as Basic Right, en J. WALDROn, Torture, Terror and Trade-Offs, en particular p. 168, con la alusión a los usos del argumento hechos por la Administración Bush tras el 11 de septiembre.

53 Así, J. Waldron, Torture, Terror, and Trade-Offs, op. cit., 177. 
próximo ataque terrorista; cuando este se produzca, aparecerán ineptos para cambiar las reacciones cuasi-pavlovianas de los gobiernos y los legislativos propensos a ellos ${ }^{54}$. Pero sobre todo, como se dijo al inicio, los argumentos de WALDRON no se dirigen a los sujetos institucionales que hasta ahora se han mostrado más dispuestos a acogerlos: las Cortes supremas, constitucionales e internacionales. En conclusión, la estrategia política de WALDRON se vuelve más eficaz si se dirige también a los jueces, como veremos enseguida.

\subsection{Una respuesta (también) jurídica}

Una respuesta jurídica a las derivaciones de seguridad puede venir del nuevo constitucionalismo ${ }^{55}$. Ciertamente, el old o ancient constitutionalism, ya reconocía los derechos al hábeas corpus en el $\$ 39$ de la Carta Magna (1215). Sin embargo, asumía que toda la esfera del gubernaculum - la prerrogativa del monarca, los poderes políticos hoy pasados a los ejecutivos- no esté sometida a los mismos controles jurídicos de los que era pasible, en cambio, la esfera de la iurisdictio ${ }^{56}$. Sin embargo, la constitución federal estadounidense (1797), antepasada del new constitutionalism, prevé la suspensión del hábeas corpus por razones de safety ${ }^{57}$ abriendo así el camino a los controles jurídicos.

En esta sub-sección se examinan, precisamente, los remedios provistos por el nuevo constitucionalismo a tres posibles conflictos entre libertad-seguridad individual, de un lado, y tres tipos distintos de seguridad, del otro. Ante todo, las libertades individuales pueden entrar en conflicto con la seguridad individual, luego con la seguridad social, finalmente con la seguridad nacional. Este último permanece como el caso paradigmático de conflicto entre libertad y seguridad, pero la posibilidad de concebir la seguridad como derecho individual y la oposición entre derechos individuales e intereses colectivos, provee un criterio para evitar balances indiscriminados.

Al conflicto se aplicará el principio de proporcionalidad, uno de los instrumentos más incisivos elaborado por el nuevo constitucionalismo global ${ }^{58}$. En otros términos, de las medidas limitativas de la libertad-seguridad individual tomadas en nombre de la seguridad colectiva se preguntará si responden a las exigencias de idoneidad, necesidad y proporcionalidad en sentido estricto respecto al fin que se proponen. Se dará, en cambio, por admitido que superan el primer test de legitimidad: ¿`son fines consti-

54 Cfr. E. A. Posner y A. Vermeulen, Terror in the Balance. Security, Liberty and the Court, Oxford, Oxford U. P., 2007: quizás los mayores partidarios de una concentración en el ejecutivo de todos los poderes de emergencia.

55 Cfr. en particular M. Rosenfeld, Judicial Balancing in Times of Stress, op. cit., quizás el tratamiento más sistemático del tema desde el punto de vista del nuevo constitucionalismo.

56 La referencia es a la clásica reconstrucción de Charles MacIllwain, Constitutionalism: Ancient and Modern, Ithaca (N. Y.), Cornell U. P., 1940.

57 Así el art. 1, inciso 9, numeral 2: «The Privilege of the Writ of Habeas Corpus shall not be suspended, unless when in Cases of Rebellion or Invasion the public Safety may require it».

58 Elaborado por la jurisprudencia administrativa y luego constitucional alemana, es mencionado por el Conseil Constitutionnel francés en la decisión sobre el état de urgence del 22 de diciembre de 2015. Cfr. Conseil constitutionnel, Décision n²015-527 QPC, 22 décembre 2015, 6: «le juge administratif est chargé de s'assurer que cette mesure [la residencia forzosa] est adapté, nécessaire et proportionnée à la finalité qu'elle poursuit». 
tucionalmente legítimos aquellos invocados? Es decir, se admitirá que la seguridad es siempre un fin legítimo, contemplado explícita o implícitamente en las constituciones.

\subsubsection{Seguridad individual}

La seguridad individual es el valor tutelado por el derecho, el bien jurídico por antonomasia, cuya garantía constituye la primera, principal e indispensable función del Estado. Hemos visto, en cambio, en 4.2.1, que la seguridad individual coincide, en su núcleo esencial, con la libertad individual. Es decir, los derechos de libertad-seguridad no son solo conmensurables sino que son precisamente el mismo derecho: cuando se viola la libertad individual se viola también la seguridad individual, y viceversa. Así, muchos argumentos a favor de la seguridad colectiva parecen persuasivos pero solo porque confunden la seguridad individual y la colectiva, absorbiendo la primera en la segunda.

En cambio, no se pueden excluir conflictos inter-rigths —entre libertad-seguridad individual y seguridad como interés colectivo y bien público- y tampoco conflictos intra-right, es decir, entre los mismos derechos a la libertad-seguridad de los que son titulares distintos individuos ( $c f r$. 4.2.3). Aquí adquiere parcial legitimidad uno de los argumentos de la retórica de la seguridad: la oposición entre derechos de los autores de los atentados y derechos de sus posibles víctimas. Entonces, conflictos intra-right entre libertad-seguridad de personas de carne y hueso, pueden efectivamente exigir elecciones trágicas típicas del pluralismo de los valores.

Es el caso de la ticking bomb: ¿es lícito torturar a un terrorista para salvar vidas humanas? ${ }^{59}$ Aplicando los tests de proporcionalidad, si la tortura se revelase idónea para salvar, necesaria a falta de otros medios menos lesivos, y proporcional respecto a los derechos implicados (integridad física del terrorista, vida de las posibles víctimas), ella podría resultar lícita. Se trata, sin embargo, como se ha señalado a menudo, de una hipótesis de escuela. En efecto, precisamente para excluir todo balance de este tipo, la Convención de 1984 prohíbe absolutamente la tortura, es decir, sin alguna posible excepción ${ }^{60}$.

\subsubsection{Seguridad social}

«Seguridad» es también usada para la seguridad social: interés colectivo o bien público tutelado sobre todo por las versiones continentales del new constitutionalism. La seguridad social es, a menudo, garantizada por las declaraciones de los derechos atribuyendo a individuos o a categorías de sujetos derechos denominados sociales, es decir, a servicios por parte de la colectividad: salud, previsiones, asistencia, a veces

59 Sobre la cuestión $c f r$., al menos, M. LA TORRE y M. LALATTA COSTERBOSA, Legalizzare la tortura? Ascesa $e$ declino dello Stato di diritto, Bologna, Il Mulino, 2013, y por último, M. LALATTA CosTERBosa (coord.), «Il silenzio della tortura», parte monográfica de la Rivista di filosofia del diritto, 2015/2.

${ }_{60} \mathrm{Cfr}$. el tenor literal de la disposición (art. 2.2 de la Convention against Torture [...], UNTS, 1984, vol. 1465, 85): «No exceptional circumstances whatsoever, whether a state of war or a threat of war, internal political instability or any other public emergency, may be invoked as a justification of torture». 
renta mínima, otras veces casa o trabajo. La seguridad social sería extraña al tema de este ensayo si no presentase cuatro analogías con la seguridad nacional, la primera de ellas, la ambigüedad individual/colectivo.

En algunos casos, «derechos sociales» indica verdaderos y propios derechos individuales, atribuidos por principios regulativos y, por tanto, justiciables como si fuesen atribuidos por reglas. En este sentido, si tengo un derecho a la salud debo ser curado, y puedo dirigirme a un juez para conseguirlo, precisamente como para cualquier otro derecho individual. En otros casos, en cambio, los derechos sociales son simples intereses colectivos, atribuidos por principios directivos, es decir, no justiciables sino que solo se actúan por parte del legislador. Es el caso del denominado derecho al trabajo que indica, a lo sumo, el objetivo del pleno empleo ${ }^{61}$.

El segundo aspecto común es que la seguridad, social y nacional, es cuestión de grado: su mayor o menor tutela depende de la política, no del derecho. El tercer aspecto es que ambas justifican la (auto)atribución a los gobiernos de poderes excepcionales: la seguridad nacional justificó la suspensión del Hábeas corpus por parte de Abraham LinCOLn durante la Guerra de Secesión, la seguridad social justificó el Nuevo Deal de Franklin D. Roosvelt después de la Gran Depresión. El cuarto y más importante aspecto común, es que ambas pueden prevalecer sobre la libertad-seguridad individual como pre-condición de ella pero, cuidado, solo hasta donde la propia pre-condición corra el riesgo de verse afectada.

Es cierto que por debajo de un nivel mínimo de seguridad, social o nacional, la tutela de cualquier derecho se haría imposible. Pero en cualquier otra situación menos extrema, distinta de la caída de la población por debajo del nivel de supervivencia o de la disolución del Estado ${ }^{62}$, la tutela de la seguridad colectiva permanece como una condición solo necesaria, no suficiente, de la tutela de los derechos individuales ${ }^{63}$. En suma, para sacrificar libertades-seguridades individuales al interés colectivo a la seguridad, superando la presunción liberal (cfr. 4.2.2) a favor de los derechos individuales, hace falta mostrar que, de otro modo, se corre el riesgo del hambre o de la anarquía.

\subsubsection{Seguridad nacional}

Finalmente, la seguridad nacional (pero también internacional o global) es el interés colectivo más invocado para limitar la libertad-seguridad. Sin embargo, para poner la cuestión sobre el terreno constitucional, en términos de principios sujetos a balance por parte de cortes supremas, constitucionales o internacionales, es necesario afrontar primero una suerte de trilema de la seguridad. O la seguridad nacional es solo un valor

61 Esta diferencia estructural se advierte en L. FERRAJOLI, Los derechos y sus garantías. Conversación con Mauro Barberis (2013), trad. cast. Madrid, Trotta, 2015. Similares distinciones son trazadas por Ronald DwORKIN entre principles y policies, y por Manolo ATIENZA y Juan RUIZ MANERO entre principios y directrices.

62 Es este el muy fuerte argumento, usado por Lord HoFFMAN en A/FC, et al. V. Secretary of for the Home Department (2004), UKHL56 (House of Lord), uno de los más famosos procesos británicos por terrorismo, $\$$ 96: «Terrorist violence, serious as it is, does not threaten our institution of government or our existence as a civil community».

63 Así, en términos de condiciones necesarias y suficientes, B. Williams, «Realism and Moralism in Political Argument», trad. it. «Realismo e moralismo in teoria politica», en id., In principio era l'azione, op. cit., 6. 
político o militar ajeno al derecho, o es siempre un principio jurídico, tan absoluto que obliga a los jueces a hacerlo prevalecer sobre cualquier otro principio, o bien es solo uno entre otros principios supremos, ponderable con otros.

4.3.3.1. Ante todo, la seguridad nacional podría considerarse un valor pre-constitucional sustraído a cualquier balance con principios constitucionales. Así podría interpretarse el venerable salus reipublicae suprema lex esto, la distinción paleo-constitucionalista gubernaculum/iurisdictio, las diversas doctrinas judiciales de la political question, o la doctrina de la soberanía de SCHMIT como decisión sobre el estado de excepción. El punto es, en cambio, que si la cuestión del conflicto entre seguridad nacional y principios constitucionales es, de hecho, llevada ante un juez, deberá encontrar respuesta en términos constitucionales, pasando así al segundo cuerno del trilema.

4.3.3.2. La seguridad nacional podría considerarse entonces un principio constitucional pero que siempre prevalece sobre otros principios constitucionales. Así podría interpretarse «lex» en salus reipublicae, la distinción paleo-constitucionalista gubernaculum/iurisdictio, la doctrina de la political question, la propia doctrina schmittiana: la seguridad nacional no sería un sino el principio constitucional supremo que prevalece siempre sobre todos los otros. Jueces internos más deferentes hacia los ejecutivos nacionales a menudo contestan precisamente así: la seguridad nacional prevalece sin más, sin consideraciones de otros principios jurídicos.

Piénsese en el caso El-Masri, ciudadano alemán de origen libanés, arrestado por error en Macedonia, remitido a los servicios secretos estadounidenses, llevado a Afganistán y torturado, y finalmente puesto en libertad; las cortes estadounidenses han rechazado sus recursos, y la Corte Suprema ha rechazado ocuparse de su caso. O bien el caso Abu Omar, imán sospechoso de terrorismo secuestrado en Milán por los servicios estadounidenses e italianos, torturado en Egipto; aquí los jueces ordinarios quisieron indagar pero la Corte constitucional los obstaculizó. En ambos casos, las razones de los malaventurados solo han sido reconocidas por la Corte europea de los derechos del hombre (cfr. 4.3.3.3).

Una teoría realista de la interpretación constitucional, que admita el carácter supremo de los principios predominantes en el balance ( $c f r .3 .4)$ debería llegar justamente a esta conclusión a partir de las decisiones de los jueces internos. Evidentemente, tenemos un candidato mejor que la dignidad humana para el rango de único principio supremo o de principio supraconstitucional: la seguridad colectiva (nacional, internacional, global) ${ }^{64}$. Pero quizás, como veremos enseguida, para establecer jerarquías axiológicas más confiables entre los principios constitucionales es necesario considerar también los balances realizados por los jueces internacionales.

4.3.3.3. La seguridad nacional, finalmente, puede considerarse un principio no más supremo que otros y, de hecho, sujeto a balances, a secas o con armonizaciones, con los derechos de libertad-seguridad ${ }^{65}$. Con la transformación del terrorismo inter-

${ }^{64}$ Cfr. G. Cerina Feroni y G. Morbidelli, «La sicurezza: un valore superprimario», en Percorsi costituzionali, 2008, 1, 31-44, con las críticas de A. VEDASCHI, «Has the Balancing of Rights Given Way to a Hierarchy of Values?», en Comparative Law Review, 1, 2010, 1-40.

${ }_{65}$ Cfr. M. Rosenfeld, Judicial Balancing in Times of Stress, op. cit., y A. VeDAschi, The Dark Side of Counter-Terrorism: Arcana Imperii and Salus Reipublicae (2015, inédito). 
nacional en fenómeno casi fisiológico, ya las cortes internas, más deferentes hacia el ejecutivo y/o hacia el legislativo controlado por el primero, parecen menos dispuestas a conceder «cheques en blanco» a los gobiernos ${ }^{66} \mathrm{y}$ a reconsiderar las relaciones libertad-seguridad. Pero esta tendencia aparece aún más clara en la jurisprudencia de las cortes internacionales que no tienen razones de deferencia hacia los ejecutivos nacionales.

Una teoría realista de la interpretación constitucional podría llegar precisamente a esta conclusión a partir de las dos decisiones de la Corte europea de los derechos del hombre en los casos El-Masri y Abu Omar. La sentencia El-Masri (2012) ha condenado a Macedonia, miembro del Consejo de Europa, por las torturas padecidas por el ElMasri por parte de los servicios de los EEUU, país ajeno al Consejo ${ }^{67}$. Análogamente, la muy reciente sentencia Nasr et Ghali (2016) ha condenado a Italia, quizás no por las torturas padecidas por Abu Omar en Egipto sino por la connivencia mostrada por las autoridades supremas republicanas hacia los servicios ocultos aliados ${ }^{68}$.

Esta conclusión, sin embargo, sería solo aparentemente realista. En efecto, las decisiones de las Cortes internacionales solo vinculan a los países firmantes de los tratados y tienen efectos poco más que simbólicos. Solo cuando las cortes internas balancean a favor de la libertad — como ha hecho la Corte Suprema estadounidense reconociendo los derechos de Hábeas corpus a los extranjeros presos en Guantánamo ${ }^{69}$ - entonces los legislativos nacionales piensan en anular los efectos de sus decisiones. En suma, los únicos jueces que producen decisiones efectivas son aquellos internos que seguirán interviniendo solo si son llamados a tales casos aunque, a lo mejor, deshaciéndose de la cuestión con el pretexto de que los controles deben ser ejercidos por los Parlamentos $^{70}$.

Sin embargo, si se admite, en los términos del tercer cuerno del dilema, que estos problemas deben encontrar soluciones constitucionales balanceadas, respetuosas de todos los valores en juego, no se puede rehuir de las siguientes interrogantes, formuladas en términos de proporcionalidad. ¿Restringir libertad y seguridad individual es siempre idóneo para incrementar la seguridad de los Estados? ¿Es siempre necesario, no sustituible con medidas menos lesivas? ¿Es, finalmente, siempre proporcionado respecto a los derechos humanos o bien vale la presunción liberal contra el sacrificio de derechos individuales a intereses colectivos? Para un jurista, estas deberían ser preguntas retóricas.

66 La metáfora es usada por el juez de la Corte Suprema Sandra Day O' ConNor en el caso Hamdi v. Rumsfeld (2004): «A state of war is not a blank check for the president when it comes to the rights of the nation's citizens».

67 Así EctHR, Grand Chambre, El-Masriv v the Former Yugoslav Republic of Macedonia, 13 de diciembre de 2012, disponible enm hudoc.echr.coe.int, que habla también de un derecho a la verdad: cfr. A. VEDASCHI, Globalization of Human Rights and Mutual Influence between Courts. The Innovative Reverse Path of the Right to the Truth, en S. SHEETRET (ed.), The Culture of Judicial Independence. Rule of Law and World Peace, Brill Nijhoff, Leiden-Boston, 2016, 107-133.

68 Así ECHR, Quatrième Section, Nasr et Ghali (Abu Omar) v. Italia, 23.2.2016, igualmente en budoc. echr.coe.int.

69 Pienso particularmente en Rasul v. Bush 542 U. S. 466 (2004), a pesar del dissenting opinion del fallecido Antonin SCALIA.

70 Como lo destaca, proveyendo una amplia casuística, A. VEDASCHI, Has the Balancing of Rights, op. cit., $23-24$. 


\section{CONCLUSIÓN}

Entonces, los pretendidos balances entre libertad y seguridad se reducen a muy poco. Los derechos individuales a la libertad-seguridad se atemperan entre sí, pero respetando prohibiciones absolutas como aquella contra la tortura. El interés colectivo a la seguridad nacional es ciertamente tutelado pero, si hay límites constitucionales al arbitrio de los gobiernos, entonces los derechos de libertad-seguridad individuales no pueden ser sacrificados a un interés colectivo en formas no idóneas, no necesarias y desproporcionadas al logro del objetivo. No pueden porque no se debe pero, más aún, porque no sirve. A menudo, serviría más silbar en la oscuridad esperando que pase la noche. 